Musées, Patrimoine et Culture scientifiques et techniques

$108 \mid 2006$

novembre-décembre 2006

\title{
Les planétariums : des musées scientifiques en effervescence
}

Pierre Lacombe

\section{OpenEdition \\ Journals}

Édition électronique

URL : http://journals.openedition.org/ocim/1325

DOI : $10.4000 /$ ocim. 1325

ISSN : 2108-646X

Éditeur

OCIM

Édition imprimée

Date de publication : 25 novembre 2006

ISSN : 0994-1908

Référence électronique

Pierre Lacombe, «Les planétariums : des musées scientifiques en effervescence », La Lettre de l'OCIM [En ligne], 108 | 2006, mis en ligne le 12 mars 2014, consulté le 20 avril 2019. URL : http:// journals.openedition.org/ocim/1325; DOI : 10.4000/ocim.1325 


\title{
Les planétariums : des musées scientifiques en effervescence
}

\author{
Pierre Lacombe *
}

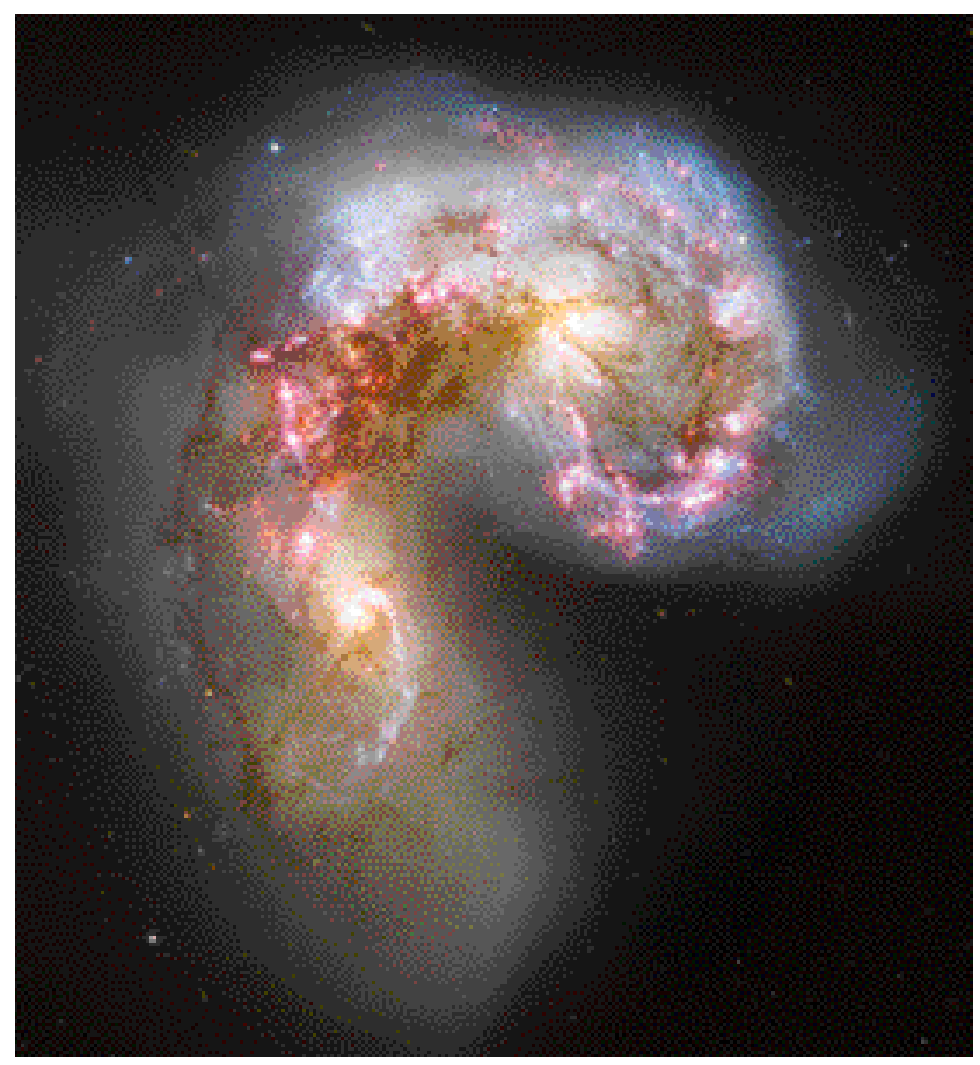

Photographie de la région centrale du groupe de galaxies en interaction appelé les Antennes obtenue par le télescope spatial Hubble. Les noyaux des deux galaxies montrent une activité intense de formation d'étoiles. (c) NASA, ESA et Hubble Heritage Team (STSCI/AURA)-ESA/Hubble Collaboration

* Pierre Lacombe est directeur du planétarium de Montréal placombe@ville.montreal.qc.ca
Responsable du planétarium de Montréal, l'auteur apporte ici le point de vue nord américain sur l'évolution exponentielle des innovations technologiques constatée depuis une dizaine d'années, les conséquences de cet avènement de l'ère du numérique sur la gestion et l'organisation des établissements ainsi que son impact sur le renouvellement et la qualité des productions désormais proposées au public.

La scène est à couper le souffle ! Une grande galaxie spirale en rotation très lente flotte dans l'Espace, lorsqu'à l'horizon de notre champ de vision, apparaît une autre galaxie spirale dont la course semble se diriger tout droit vers la première. Peu à peu, les mouvements de certaines étoiles des deux galaxies se font plus accélérés et on voit se former des ponts de matière en mouvement continu entre les deux galaxies. La collision semble inévitable et bien qu'elles se rencontrent, les deux galaxies poursuivent leur chemin respectif. Leur apparence a cependant bien changé et, ici et là, on voit apparaître des régions de formation d'étoiles. Un nouveau cycle débute.

Nous venons d'assister en accéléré au ballet galactique que joueront entre elles, notre Voie Lactée et la galaxie d'Andromède, dans plusieurs millions d'années. La simulation est saisissante et seules les nouvelles technologies de vidéo numérique et les capacités de calcul des récents microprocesseurs permettent d'obtenir un tel réalisme. Bienvenue dans le planétarium du XXI ${ }^{\mathrm{e}}$ siècle. 


\section{L'origine des planétoriums modernes}

Dès l'Antiquité, et dans le but de mieux comprendre leur univers, de nombreux philosophes et astronomes tentent de reproduire la voûte céleste et de simuler les mouvements de certains astres parmi les étoiles. De par leur simplicité, leurs premiers modèles sont inadéquats ; la cartographie céleste est trop imprécise et les technologies d'horlogerie et d'optique sont à peu près inexistantes. Les années passent et les astronomes améliorent peu à peu leurs instruments scientifiques et développent des outils théoriques de plus en plus sophistiqués. Le développement des lunettes méridiennes et des horloges astronomiques a ainsi permis aux astronomes de dresser des catalogues d'étoiles de plus en plus complets et précis et aux artisans de confectionner des atlas et globes célestes d'une rare beauté. L'atlas céleste Uranographia de Johann Bode (1747-1826) publié en 1801 et le globe céleste de Vincenzo Coronelli (1650-1718) réalisé entre 1681 et 1683 pour le roi de France Louis XIV sont d'excellents exemples de chefs-d'œuvre de précision et de rigueur scientifique qui synthétisent les connaissances astronomiques et cartographiques de leur époque.

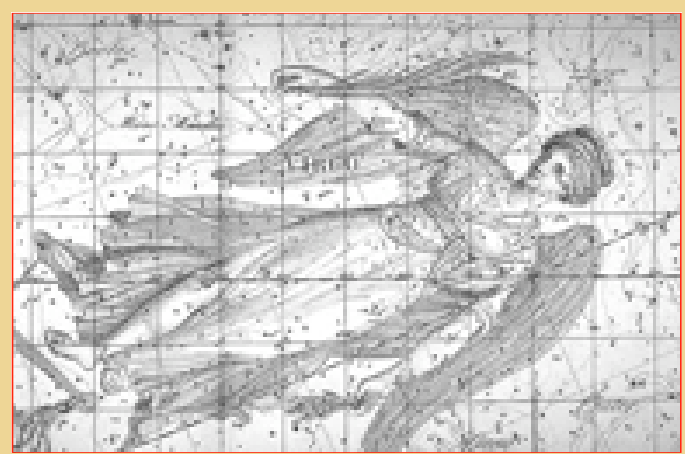

La constellation de la Vierge extraite de l'atlas céleste Uranographia publié en 1801 par Johann Bode. Cet atlas, en plus de présenter les positions précises de plus de 17000 étoiles, indique aussi les positions de plus de 2500 nébuleuses découvertes et cataloguées par William Herschel.

() The Linda Hall Library of Science, Engineering and Technology

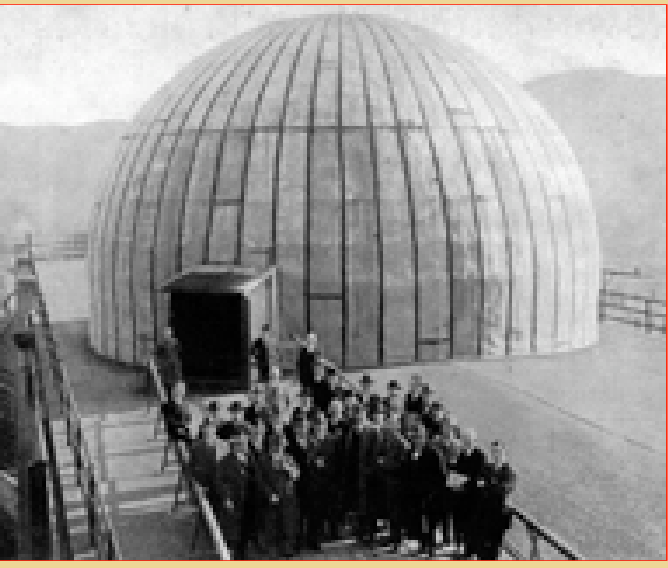

Le premier planétarium du monde installé sur le toit de l'entreprise Zeiss à Jena en 1923.

$C^{\prime}$ 'est à cet endroit que l'on teste et améliore le premier planétaire inventé par Walter Bauersfeld. (๖) Carl Zeiss Jena

mécanismes capables de simuler les mouvements des planètes autour du Soleil. Ces instruments, appelés planétaires, sont devenus des outils pédagogiques très populaires et des objets d'art très prisés par les collectionneurs.

Il faut attendre le début du $X^{e}$ siècle pour que la fusion des deux modèles se réalise enfin. Lidée novatrice émerge dans l'esprit d'un jeune ingénieur allemand, Walter Bauersfeld (1879-1959) à l'emploi de la société Zeiss à Jena : mettre à profit la technologie de la projection optique. En août 1923, le premier planétaire moderne est installé et testé sous un dôme géodésique temporaire de 16 mètres de diamètre. L'expérience s'avère un énorme succès et les foules accourent pour contempler simultanément la voûte étoilée, le Soleil, la Lune et les planètes visibles à l'œil nu ainsi que leurs mouvements apparents. L'enthousiasme pour cette innovation est tel que de nombreuses villes européennes se dotent alors d'un planétarium : Berlin en 1925, Vienne en 1927, Rome en 1928, Moscou en 1929. Les villes nord-américaines tombent elles aussi rapidement sous le charme de cette nouvelle technologie : Chicago en 1930, Philadelphie en 1933, Los Angeles et

Pour observer le ciel étoilé tel qu'il apparaît dans la réalité d'un observateur terrestre, il faut cependant changer de point de vue et se représenter la voûte céleste vue de l'intérieur. De nombreux artisans ont donc construit des globes géants dans lesquels des visiteurs pouvaient observer le ciel étoilé et certains mouvements de la sphère céleste. Un modèle de ce genre existe toujours au planétarium Adler à Chicago : la sphère dite d'Atwood de 4,6 mètres de diamètre construite en 1913.

Parallèlement, une meilleure compréhension des mouvements planétaires grâce à la théorie de la gravitation d'Isaac Newton (1642-1727) et le développement de la technologie des outils de précision ont permis de construire des
New York en 1935 et Pittsburgh en 1939. C'est en 1937 que le premier planétarium apparaît temporairement à Paris lors de l'ouverture du Palais de la Découverte dans le cadre de l'Exposition Universelle.

Au fil des années, les planétariums ont vu leur popularité et leur nombre augmenter régulièrement pour atteindre un sommet à la fin des années soixante avec l'engouement extraordinaire du public pour la conquête de la Lune. Une croissance plus lente se poursuit toujours aujourd'hui, mais les énergies sont principalement dirigées maintenant vers la révolution numérique et les nouvelles technologies de productions multimédias. 
Depuis 10 ans déjà, la communauté des planétariums vit un changement organisationnel continu, comparable et aussi important que celui vécu par les professionnels du domaine du secrétariat à l'arrivée des micro-ordinateurs qui a relégué au musée, et ce presque instantanément, les vieilles machines à écrire électriques. Le rythme des innovations technologiques est effréné et chaque année, de nouveaux produits ou des améliorations importantes sont dévoilés aux spécialistes des planétariums. Le marché est en pleine croissance et les entreprises dont les services sont dédiés principalement aux planétariums se multiplient.

Il devient donc difficile, pour les gestionnaires et les professionnels de ces institutions muséales scientifiques de comparer les plates-formes technologiques offertes et de faire le meilleur choix pour améliorer la qualité de leurs produits éducatifs et grand public.

\section{Une révolution technologique}

Depuis l'invention du premier planétarium moderne en 1923 et jusqu'à la fin des années quatre-vingt, les professionnels des planétariums ont consacré beaucoup d'énergie à améliorer les séances offertes aux visiteurs dans le théâtre des étoiles des institutions. Peu à peu, on ajoute des images et des effets visuels spéciaux pour mieux présenter et expliquer des phénomènes astronomiques, tels les éclipses et les étoiles filantes. Mais, comme le souligne de nombreux auteurs, les séances demeurent toujours des conférences illustrées sous les étoiles.

Devant la disponibilité et la prolifération des images astronomiques, les présentations deviennent au fil des années visuellement très élaborées. On ajoute d'autres projecteurs diapositives et d'effets spéciaux et superpose le tout de compositions musicales originales. La composante spectacle des séances sous la voûte étoilée prend alors une importance croissante.

Les développements de l'informatique et des capacités de calcul des microprocesseurs accélèrent cette évolution. Les systèmes de contrôle informatisé font leur apparition dans les planétariums et il n'est pas rare de voir des installations permanentes de plusieurs dizaines de projecteurs de toutes sortes dans les théâtres des étoiles. L'animateur, autrefois présent dans cette salle,

\section{Le planétorium de Montréal \\ Un oasis francophone \\ en Amérique du Nord}

Il y a un peu plus de quarante ans, le $1^{\text {er }}$ avril 1966, le planétarium de Montréal ouvrait ses portes au public. L'événement était d'importance : pour la première fois au Canada, un grand planétarium mettait le ciel étoilé à portée de compréhension du public, à portée d'expérience individuelle... La réalité virtuelle bien avant la lettre!

Depuis son ouverture, ce sont plus de 6 millions de visiteurs qui ont pu s'initier aux secrets et aux merveilles de l'Univers. De tels résultats sont liés fondamentalement à sa mission d'éducation. Le planétarium de Montréal n’a cessé, par des productions multimédias originales et de multiples activités, de vivre au rythme des grands événements astronomiques, de faire écho aux recherches scientifiques et de vulgariser les connaissances. De plus, une équipe d'astronomes professionnels et d'animateurs passionnés présente des séances en astronomie dans le théâtre des étoiles, conçoit des expositions thématiques, offre des cours sur la pratique de l'astronomie, rédige des chroniques dans les journaux, répond aux questions du public et maintient un site Internet de qualité... Tout cela pour

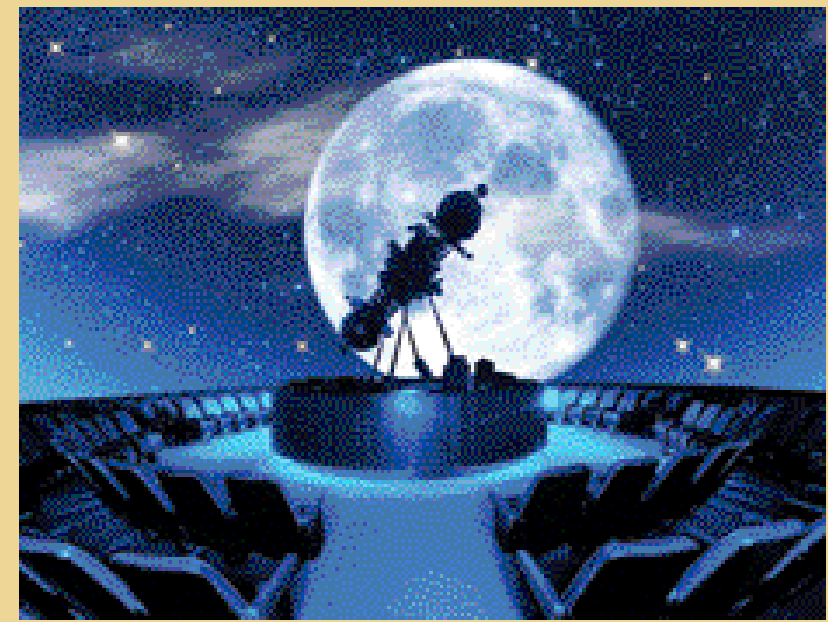

Montage photographique présentant la pleine lune et le ciel étoilé au planétarium de Montréal. (c) Planétarium de Montréal/Sophie Desrosiers

mieux faire connaître le monde de l'Astronomie au plus grand nombre de personnes.

40 ans plus tard, la mission du Planétarium, énoncée par son fondateur, Pierre Gendron, n'a rien perdu de son actualité ni de sa nécessité : rendre l'Astronomie accessible à tous. En renouvelant fréquemment sa programmation et en commentant les spectacles que l'Univers nous offre, le planétarium de Montréal souhaite donc partager, longtemps encore, sa passion de l'Astronomie. 
cède peu à peu sa place à une narration préenregistrée. Ce changement est vécu difficilement par de nombreux professionnels des planétariums tandis que d'autres répondent avec enthousiasme à la nouveauté. La réponse du public est ambiguë, mais les fréquentations qui avaient chutées à la fin du programme d'exploration lunaire sont cependant à la hausse. L'espoir renaît.

Malheureusement, devant la quantité et la qualité des effets visuels produits par le milieu cinématographique en particulier et devant les exigences du public, il devient vite évident que les productions multimédias des planétariums ne peuvent soutenir la comparaison. Deux innovations apparaissent alors dans les théâtres des étoiles. D'abord, les projecteurs diapositives laissent de plus en plus de place aux vidéoprojecteurs et ensuite, on tente par des images fixes de plonger les spectateurs dans un environnement immersif.

Un équilibre semble trouvé auprès des visiteurs et des professionnels ; dans de nombreux planétariums, à la production multimédia thématique s'ajoute en deuxième partie une balade sous les étoiles conduite par un animateur. Le taux de satisfaction des visiteurs est élevé.

Mais une révolution technologique se prépare, car on constate rapidement que le couplage de la technologie vidéo à l'approche immersive donnera des résultats visuels époustouflants. Imaginez-vous non seulement observer le ciel étoilé mais de vous y déplacer pour y repérer certains objets célestes et les étudier en trois dimensions. Après de multiples balbutiements technologiques, le premier système vidéo pouvant à la fois présenter un ciel étoilé et de multiples images sous un dôme de planétarium voit le jour à la fin des années quatre-vingt-dix. Depuis, d'autres systèmes similaires ont été développés et aujourd'hui, c'est plus de 200 planétariums dans le monde qui se sont convertis à l'ère du numérique.

\section{Une renaissance publique}

Les nouvelles possibilités de simulation qui s'offrent aux astronomes et aux professionnels des planétariums sont désormais presque infinies. Comment le public réagira-t-il à ces changements ? Force est de constater que la réaction de celui-ci jusqu'à maintenant est presque excessive et qu’à certains endroits, on frise un peu la folie.

Cette renaissance des planétariums débute en l'an 2000, en cette période de transition vers le nouveau millénaire. Le planétarium de New York, pour ne pas

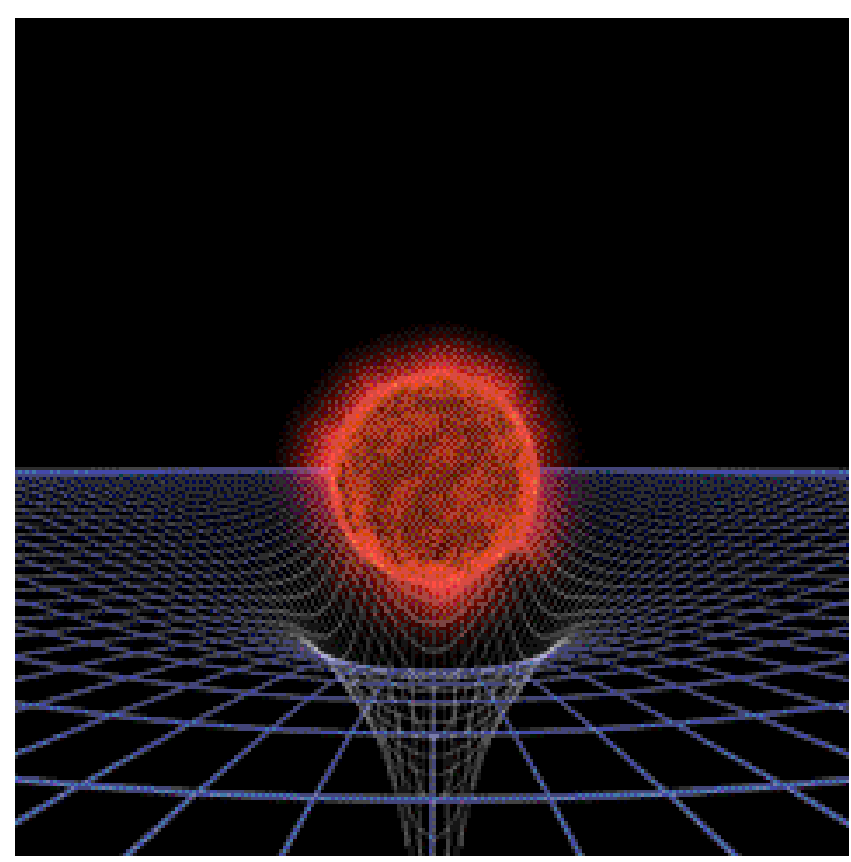

Extrait d'une simulation présentant la déformation de l'espace-temps en présence d'un objet massif.

Ce concept est essentiel pour bien comprendre la théorie de la relativité d'Einstein et l'origine des trous noirs.

(c) Denver Museum of Nature \& Science

le nommer, renaît alors littéralement de ses cendres et renouvelle complètement la perception du public envers les planétariums. Non seulement il met à profit l'immersion des visiteurs, mais développe une rigueur exemplaire dans la justesse scientifique des visualisations.

Les attentes du milieu des planétariums et du public étaient élevées face aux changements proposés et le succès de New York a permis de catalyser le développement des planétariums vers un avenir prometteur.

Depuis, les succès se multiplient et des projets de rénovation ou de nouveaux planétariums se réalisent chaque année. Une effervescence a gagné la communauté et nombreux sont ceux qui espèrent que leur institution se convertira bientôt aux technologies numériques. Certains demeurent encore sceptiques et sont inquiets que l'aspect spectaculaire de l'immersion et de la visualisation mette de côté la qualité du message scientifique. Le danger existe mais pour les optimistes il ne faut pas confondre l'outil et l'usage que l'on peut en faire. De toute façon, même pour les planétariums dits traditionnels, la tentation est toujours présente de miser sur les effets visuels et les belles images astronomiques aux dépens du contenu scientifique. La prudence sera donc toujours de mise et demeurera la responsabilité des directions des planétariums. 
Il ne faudrait pas croire que la technologie numérique n'est disponible que pour les planétariums possédant un grand théâtre des étoiles et beaucoup d'employés spécialisés. Depuis peu, des vidéoprojecteurs équipés de lentilles spéciales peuvent être utilisés dans des théâtres de petites dimensions. En fait, avec un seul de ces vidéoprojecteurs, la technologie numérique immersive est maintenant offerte aux visiteurs de ces institutions. Même les planétariums dits portatifs bénéficient aussi de la technologie numérique. Bref, c'est toute la communauté des planétariums qui est touchée par la révolution technologique.

Les succès futurs des nouveaux planétariums numériques reposeront non pas sur la technologie, mais sur la qualité des productions multimédias et la créativité des professionnels. Bien sûr, la technologie numérique est une aide précieuse mais elle ne demeure qu'un outil qui montre cependant un grand potentiel. Certains indices sont encourageants. Bien qu'approximatif, un sondage informel auprès des 3000 planétariums dans le monde montre que plus de 100 millions de personnes fréquentent ces institutions annuellement. Ces chiffres sont impressionnants, et confirment la hausse de fréquentation des dernières années. Le public aime la nouvelle technologie numérique dans les planétariums.

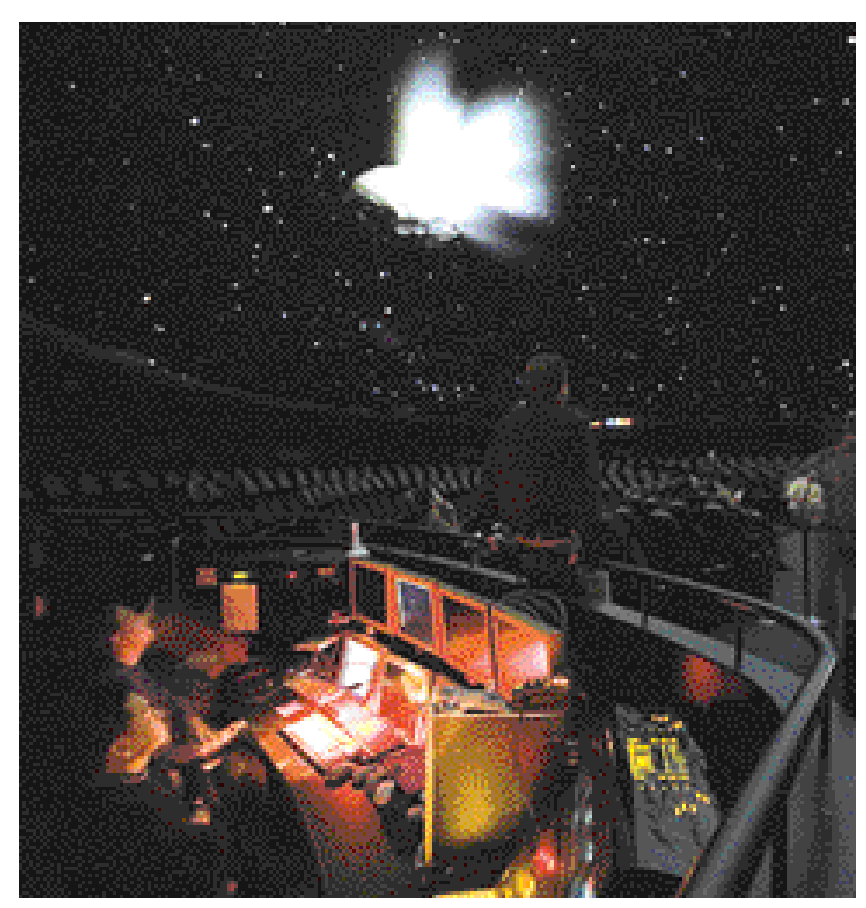

Test de simulation numérique dans le théâtre des étoiles du planétarium Hayden de New York pour le spectacle Cosmic Collisions. (c) American Museum of Natural History/Denis Finnin

\section{Tous pour un !}

Pour de nombreux intervenants du milieu, la conversion des planétariums traditionnels vers la technologie numérique est une question de temps. Temps qu'il faudra pour trouver le financement nécessaire à la rénovation des installations et des équipements. Mais attention!

La courte et récente expérience des planétariums déjà convertis au numérique démontre que les coûts de production des présentations multimédias ont aussi grimpé en flèche. Cela n'est pas surprenant lorsque l'on s'aperçoit que ces planétariums n'hésitent pas à appeler en renfort des scénaristes, des réalisateurs, des infographistes, des musiciens et des scientifiques pour la réalisation des nouvelles productions. De tels spécialistes sont souvent formés pour le nouveau médium numérique et demandent des coûts élevés de main-d’œuvre.

\section{I'International Planetorium Society}

Le nombre de planétariums croissant sans cesse au fil des décennies, les travailleurs et les intervenants du milieu ont convenu, dans les années cinquante, de l'importance de se regrouper et de se rencontrer régulièrement. On assiste donc d'abord en 1959 à la naissance d'une organisation internationale regroupant les directeurs des planétariums des grandes villes et dans les années soixante, à l'éclosion de nombreuses organisations régionales, particulièrement aux États-Unis.

L'inauguration d'un nombre élevé de planétariums à la fin des années soixante multiplie les occasions de conférences et de colloques et les éducateurs des planétariums fondent une organisation internationale en 1970. Celle-ci deviendra l'International Planetarium Society (IPS) quelques années plus tard.

Aujourd'hui, l'IPS compte près de 700 membres regroupés dans plus de 20 associations régionales ou nationales et est présente dans plus de 35 pays dans le monde. L'organisation représente les planétariums de toutes dimensions, qu'ils soient fixes ou portatifs et permet à ses membres d'échanger et de partager leurs expériences par la tenue de conférences bisannuelles et la publication d'un journal spécialisé, The Planetarian.

Au cours des dernières années, les conférences de l'IPS ont suscité un vif intérêt auprès des membres ; chacune des occasions étant un prétexte pour les fournisseurs de présenter les dernières innovations technologiques. La prochaine conférence aura lieu en juin 2008 au planétarium Adler à Chicago aux États-Unis et, déjà, on estime qu'elle sera l'une des plus fréquentées! 


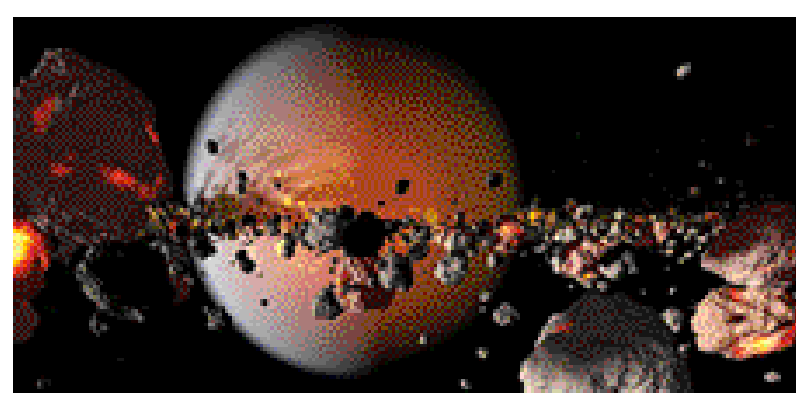

Extrait d'une simulation numérique présentant la formation de la Lune en révolution autour de la Terre. () American Museum of Natural History

Il est donc normal que les directions des planétariums soient inquiètes puisque c'est l'avenir de leur institution qui se joue si la transition technologique n'est pas bien planifiée, aussi bien en ressources financières qu'en ressources humaines. Dans ce contexte, les alliances et les consortiums entre les planétariums se multiplient pour offrir des productions multimédias de qualité et surtout pour renouveler régulièrement le produit offert aux visiteurs. Même les fournisseurs de systèmes immersifs, saisissant l'opportunité, se lancent eux aussi dans la réalisation de productions multimédias. Le défi est de taille pour satisfaire les diverses clientèles des planétariums.

Par choix ou par faute de moyens, des planétariums ne se convertiront pas complètement au numérique. Ils miseront donc sur une simulation du ciel étoilé de qualité et sur une utilisation novatrice des anciennes technologies. Les médiateurs scientifiques joueront alors un rôle très actif auprès des visiteurs et devront, comme toujours, se surpasser pour leur offrir une expérience inoubliable.

D'autres soucis s'ajoutent à l'inquiétude des responsables des planétariums tentés par le changement technologique. Que dire par exemple de la durée de vie de ces nouveaux équipements spécialisés ? Au rythme des innovations annoncées, les équipements d'aujourd'hui seront-ils désuets dans cinq ans? Que dire aussi des coûts d'entretien de ces équipements dont on parle peu? Les incertitudes sont nombreuses et le saut vers la technologie numérique peut ainsi réserver des surprises. Les planétariums sontils prêts à affronter ces changements ? Car c'est bien de cela qu'il s'agit.

\section{Un avenir prometteur}

De nombreux audacieux ont déjà saisi l'opportunité et participent activement à la renaissance des planétariums auprès du public. Des nouveaux planétariums surgissent dans des endroits inusités, comme sur un bateau de croisière par exemple, et la technologie numérique ne cesse d'évoluer et de surprendre.

Déjà, l'imagerie laser à haute définition s'implante dans les planétariums et s'apprête, à moyen terme, à remplacer les vidéoprojecteurs. On le constate, l'innovation est féconde dans le milieu des planétariums.

Il ne faudrait pas reprocher aux directions des institutions d'être, pour certaines, enthousiastes et prêtes à plonger et pour d'autres, inquiètes et patientes. Cette ambivalence permet au contraire un vrai débat au sein de la communauté et ne peut que promettre un avenir stimulant pour les planétariums et leur personnel.

Les défis sont nombreux mais la réaction des visiteurs des planétariums nous confirme encore aujourd'hui que la magie existe sous les étoiles !

\section{Bibliographie}

Chartrand, M.-R. A Fifty year anniversary of a two thousand year dream, The Planetarian, vol. 2, n³, septembre 1973, pp. 95-101.

Cooper Jr., H. S. F. The Rose Center : sphere of influence, Natural History, vol. 2, 2000, pp. 50-59.

Deans, P. Magic under the domes, Sky \& Telescope, vol. 107, ${ }^{\circ} 1$, janvier 2004, pp. 52-59.

Escalon, S. Planétariums : découvrez les spectacles du future, Ciel $\&$ Espace, n404, juillet, 2004, pp. 86-90.

Faidit, J.-M. Anniversaire : 75 ans de Planétarium moderne, Planétariums, 1998, pp. 50-52.

Faidit, J.-M. Planetarium in France, The Planetarian, vol. 35, n³, septembre 2006, pp.14-16.

Hagar, C.-F. Planetarium : window to the Universe. Oberkochen : Carl Zeiss, 1980, 193 p.

Matthews, M. Digital domes and the future of Planetariums, The Planetarian, vol. 34, $\mathrm{n}^{\circ}$ 4, décembre 2005, pp. 16-18.

Petersen, M. What the heck is a Planetarium anymore ?, The Planetarian, vol. 34, ${ }^{\circ} 2$, juin 2005, pp. 19-21.

Piednoël, É. Panorama des planétariums en France, la Lettre de l'OCIM, n 45,1996 , pp. 8-10.

Werner, H. From the Aratus globe to the Zeiss planetarium. Stuttgart Verlag Gustav Fischer, 1957, 204 p.

Wyatt, R. Planetarium paradigm shift, The Planetarian, vol. 34, $\mathrm{n}^{\circ} 3$, septembre 2005, pp. 15-19.

Collectif, Planetarium : a challenge for educators, dir. Smith, D. et Haubold, H., New York : Nations-Unies, 1992, 150 p.

Collectif, Communicating astronomy with the public, dir. Robson, I. et Christensen, L. L., ESO-ESA-UAI, 2005, 398 p. 